\title{
Strates
}

STRATES Matériaux pour la recherche en sciences sociales

$10 \mid 2001$

Villageois et citadins de Grèce

\section{Y a-t-il un modèle grec d'exploitations agricoles ?}

\section{Hugues Lamarche}

\section{OpenEdition}

\section{Journals}

\section{Édition électronique}

URL : http://journals.openedition.org/strates/75

DOI : $10.4000 /$ strates.75

ISSN : $1777-5442$

\section{Éditeur}

Laboratoire Ladyss

Édition imprimée

Date de publication : 1 mai 2001

ISSN : 0768-8067

\section{Référence électronique}

Hugues Lamarche, "Y a-t-il un modèle grec d'exploitations agricoles ? », Strates [En ligne], 10 | 2001, mis en ligne le 20 mai 2005, consulté le 08 septembre 2020. URL : http://journals.openedition.org/ strates/75; DOI : https://doi.org/10.4000/strates.75

Ce document a été généré automatiquement le 8 septembre 2020

Tous droits réservés 


\title{
Y a-t-il un modèle grec d'exploitations agricoles?
}

\author{
Hugues Lamarche
}

1 Tenter de répondre à cette question implique de se situer d'une part dans une démarche typologique des modes de fonctionnement des exploitations agricoles (une modélisation), et d'autre part dans une approche comparative (confronter l'agriculture grecque à celle des autres pays européens).

2 Compte tenu de la très grande diversité de la population étudiée (diversité géographique, mais aussi structurelle et socioculturelle) il est nécessaire avant tout de se doter d'une grille d'analyse théorique qui permette une classification de toutes les exploitations en fonction de leur histoire, de leur situation matérielle, de leur organisation et de leur projet. En Europe, la modernisation de l'agriculture se matérialise globalement par la disparition du mode de production paysan et l'installation d'un modèle Entrepreneurial ${ }^{1}$. C'est donc autour du développement de l'intensification des systèmes de production, de l'installation d'un modèle productiviste et de l'éventuelle remise en cause de ce dernier que nous avons construit la grille théorique d'analyse présentée plus loin.

D'autre part, afin de rendre opératoire cette grille d'analyse, c'est-à-dire d'être en mesure de classer chaque exploitant dans la typologie, nous avons réalisé une enquête auprès des exploitants agricoles. Un questionnaire commun permettant de recueillir les renseignements nécessaires pour opérer une classification selon cette grille a été élaboré et passé à des exploitants individuels. L'enquête menée entre 1993 et 1995, dans le cadre du REALCEA ${ }^{2}$, dans quatre pays de l'Union européenne (Portugal, Italie, Grèce et France) et sur 16 terrains a concerné 746 exploitants. 


\begin{tabular}{llc}
\hline Lieux & & Nombre d'exploit ants enquê tés \\
\hline Italie & & 145 \\
& Piémont & 99 \\
& Vénétie & 46 \\
\hline Grèce & Vergina & 211 \\
& Thrace & 50 \\
& Thessalie & 48 \\
& Béotie & 50 \\
\cline { 2 - 3 } Portugal & Péloponè se & 43 \\
& & 20 \\
& Aveiro & 207 \\
& Evora & 44 \\
\cline { 2 - 3 } & Mafra & 39 \\
\cline { 2 - 3 } & Chaves & 40 \\
\hline France & Vil a do Conde & 41 \\
\hline & & 43 \\
& Bretagne & 47 \\
\cline { 2 - 3 } & Marais poitevin & 38 \\
& Causs es & 42 \\
\hline
\end{tabular}

4 S'est posée alors naturellement la question très délicate de l'échantillonnage. Nous avons distingué le niveau global du niveau local.

5 Au niveau global (choix des pays et des terrains), notre souci était de refléter la diversité (pays du Nord et pays du Sud et spécificités agraires régionales). Si l'échantillonnage au niveau des terrains est assez satisfaisant pour la Grèce, la France et le Portugal, on peut regretter que seule l'Italie du Nord ait été enquêtée; on peut regretter aussi le déséquilibre entre pays du Nord (représenté par un seul pays, la France) et pays du Sud de l'Europe. La présence dans l'échantillon d'un second pays caractéristique de l'Europe du Nord (la Hollande ou l'Allemagne par exemple) aurait permis un rééquilibrage.

6 L'échantillon se répartit de la façon suivante :

7 Au niveau local (choix des exploitants à enquêter), nous avons voulu travailler sur un échantillon conséquent d'exploitants prenant en compte les différentes situations locales dans lesquelles se trouvent les exploitations individuelles. Celles-ci se définissent et se différencient les unes des autres à partir de critères extrêmement variés selon les pays et surtout les régions (surface, niveau technique, organisation du travail, revenu extérieur, etc.). Face à ce problème nous avons choisi d'utiliser au mieux les connaissances et les compétences des chercheurs locaux; le choix des exploitants enquêtés a donc été effectué sur chaque terrain par un chercheur qui en avait une bonne connaissance et qui était donc en mesure de satisfaire l'exigence de construire un échantillon tenant compte de la diversité des situations de l'agriculture locale.

Nous présenterons dans un premier temps la démarche théorique et son application à partir des résultats de l'enquête et dans un second temps nous comparerons la situation des agriculteurs grecs enquêtés à celle des agriculteurs des autres pays européens étudiés.

Présentation de la démarche 
9 Nous ne présenterons pas ici tous les présupposés théoriques sur les modèles de fonctionnement des exploitations agricoles ${ }^{3}$ à partir desquels nous avons construit notre démarche. Disons simplement que celle-ci part du postulat que l'exploitation agricole s'organise principalement autour de deux logiques de fonctionnement, centrées l'une autour de la famille, l'autre autour d'un rapport d'autonomie/ dépendance, et que des modèles de fonctionnement d'exploitations agricoles peuvent être définis à partir du croisement de ces deux logiques. Les exploitations, selon leur histoire, leur structure de production et leurs aspirations, forment un objet sociologique particulièrement hétérogène ; cette diversité se matérialise, entre autres, dans les rapports que chaque exploitant entretient avec sa famille (place et rôle de la famille dans l'organisation, le fonctionnement et la reproduction de l'exploitation) et dans la nature des relations qu'il établit avec le marché et, plus généralement, avec la société globale.

Quatre modèles théoriques, schématisés dans le graphique suivant, sont ainsi définis $a$ priori:

11 1) le modèle Paysan ou Vivrier. Ce modèle se caractérise à la fois par une réalité où la famille tient une place centrale aux différents niveaux de fonctionnement de l'exploitation (aussi bien celui de l'organisation du système de production que du système de valeurs et représentations) et par une faible intégration à l'économie de marché. Modèle dominant dans les sociétés paysannes, il a été progressivement abandonné, dans les sociétés en voie de modernisation, par les agriculteurs désireux de se moderniser. Jugé traditionnel et archaïque dans les sociétés industrialisées, c'est un modèle dont la logique de fonctionnement ne permet pas de dégager un revenu suffisant pour donner accès à la société de consommation et qui, de ce fait, ne semble plus avoir sa place dans les sociétés modernes industrialisées. Cependant, dans le contexte actuel de crise économique et du travail (persistance d'un taux de chômage élevé, insécurité et flexibilité de l'emploi, réapparition et installation d'une certaine précarité et pauvreté, etc.) et de remise en cause plus ou moins profonde du productivisme, il est tout à fait envisageable qu'un tel modèle présente à nouveau un intérêt pour certaines catégories d'agriculteurs ou d'individus.

D`pend ance

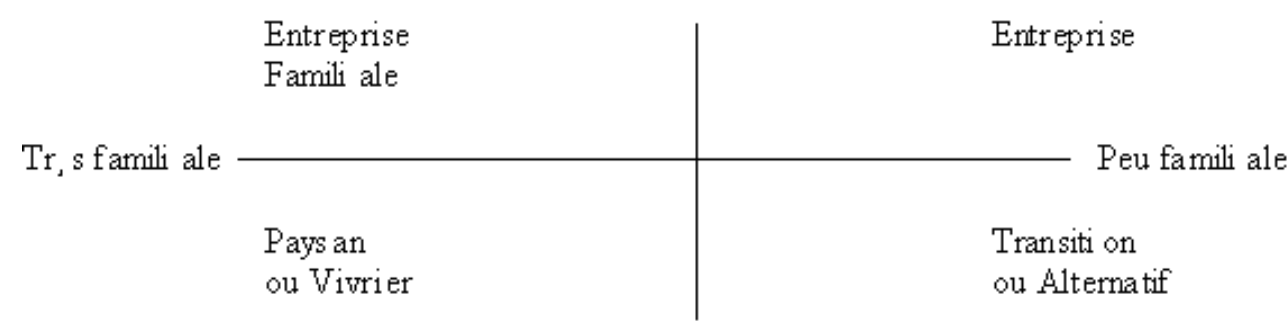

Autonomie

122 et 3) les modèles Entrepreneuriaux, modèle Entreprise ou modèle Entreprise familiale, se caractérisent par une très forte intégration au marché. C'est autour de ces deux modèles que les exploitants se sont construit un statut professionnel et social "respectable"; ces exploitants sont reconnus comme des techniciens, des chefs d'entreprise, des professionnels modernes, et c'est autour de cette élite de producteurs agricoles que s'est ancré et développé le système productiviste. Mais, fortement contestés depuis déjà quelques années, les modèles Entrepreneuriaux devront se 
transformer s'ils veulent se maintenir, car ils génèrent trop de dépendance et deviennent donc trop vulnérables; contraints à de plus en plus de dépendance (technique, économique, financière et idéologique), les exploitants sont de moins en moins capables de gérer les risques quels qu'ils soient (climatiques, financiers ou écologiques) et la fragilité qui en résulte les oblige à avoir recours à toujours plus d'assistance et donc à être encore plus dépendants. Il est clair, aujourd'hui, que la société est de moins en moins disposée à accepter toutes les contraintes liées au fonctionnement particulier de ces deux modèles ;

13 4) le modèle de Transition ou Alternatif. Une telle situation peut être interprétée comme une transition entre le modèle PaysanVivrier et les modèles Entrepreneuriaux, qu'ils soient familiaux ou non, c'est-à-dire un moment dans l'évolution de certains exploitants désireux de se moderniser; ce fut le cas dans les pays agricoles les plus intensifiés et ce peut être encore le cas dans les pays qui poursuivent un processus d'intensification de leur agriculture, fondé, entre autres, sur la transformation de la paysannerie.

14 Mais ce modèle peut être porteur aussi d'un changement plus profond correspondant aux transformations qui s'opèrent parmi les exploitants du type Entrepreneur, ceux qui remettent en cause leur mode de fonctionnement antérieur dominé par la logique productiviste. Dans ce cas, il s'agit d'une évolution en profonde rupture avec les trois autres modes de fonctionnement, parce qu'il s'organise autour d'une double tendance dont l'association nous est peu familière : perte progressive de la place prépondérante de la famille et recherche d'une plus grande autonomie dans le fonctionnement de l'exploitation.

15 En ce qui concerne la famille, cette évolution est déjà bien amorcée dans certaines sociétés où les exploitations ont connu une profonde transformation des structures familiales et de leur rôle dans le fonctionnement général de l'exploitation. La famille y tient toujours une place importante, mais celle-ci ne peut plus s'analyser de la même façon, c'est-à-dire en utilisant les mêmes critères et les mêmes définitions.

16 Pour ce qui est de la logique d'autonomie/dépendance, la volonté affirmée par certains exploitants d'une plus grande autonomie dans le fonctionnement (baisse des coûts de production et de la consommation des intrants, diversification du système de production, pluriactivité, etc.) peut s'imposer comme une tendance d'évolution lourde et difficilement contournable. Notons que cette recherche constante de plus d'autonomie s'observe aux différents niveaux du processus de production.

17 Tout ceci admis, reste la question de la définition des deux axes; à partir de quels critères et de quelles variables va-t-on classer les exploitants en fonction de leur rapport à la famille et à la dépendance ? C'est là un point important, car du niveau de précision et de finesse des variables construites pour déterminer les axes dépend la pertinence des quatre modèles définis.

18 L'axe Familial a été construit avec les quatre variables synthétiques suivantes, élaborées à partir de croisements entre diverses variables brutes :

19 - le rapport au patrimoine (PAT); il s'agit d'évaluer par cette variable le type de relations que l'exploitant entretient avec son patrimoine (terre, bâtiments, cheptel, etc.) et le degré d'attachement qu'il a vis-à-vis de lui (rapport à la propriété familiale et à la transmission de ce patrimoine) ; 
20 - l'organisation du travail (IMF) ; comment s'organise le travail sur l'exploitation? Quelle place est donnée au travail familial et au travail salarié non familial ?

21 - la reproduction de l'exploitation (RPA et LGF) ; la succession est-elle un élément important dans la prise de décision et dans les stratégies élaborées par le chef d'exploitation? ou bien l'exploitation est-elle simplement un outil de production susceptible d'être vendu le moment venu? Fonctionne-t-elle plus ou moins dans une logique familiale de reproduction de l'exploitation?

22 La mise en relation de ces différentes variables codées en deux item (faiblement familial et fortement familial) permet le positionnement des exploitants sur l'axe "Logique familiale », certains s'opposant en se situant à l'une ou l'autre des extrémités de l'axe et d'autres se situant sur des positions intermédiaires.

23 L'axe Autonomie/dépendance s'organise, lui aussi, autour de quatre variables synthétiques :

24 - la caractérisation du système de production (TEC), évaluée à partir de l'organisation des productions, le niveau technique, la productivité, etc.

25 - les stratégies d'investissements et de financement de ces investissements (FIN) : importance, nature et régularité des investissements réalisés, utilisation du crédit et de l'autofinancement, niveau d'endettement, etc.

26 - le rapport au marché (RPM), évalué par l'importance des produits vendus, le niveau d'auto-approvisionnement et d'auto-consommation, la nature et la diversité des circuits de commercialisation, etc.

27 - le rapport à la société globale et locale (RLG) ; l'exploitant organise-t-il, à la fois sur le plan professionnel et plus général du mode de vie, ses relations sociales principalement dans des réseaux localisés? Ou est-il plutôt intégré dans des réseaux plus larges et extérieurs à la société locale?

28 Comme précédemment, la mise en relation de ces différentes variables codées en deux item (faiblement dépendant et fortement dépendant) permet le positionnement des exploitants sur l'axe " Autonomie/dépendance ", certains, très dépendants, s'opposant sur l'axe aux très autonomes, d'autres se positionnant entre ces deux extrêmes.

La mise en correspondance de ces différentes variables synthétiques (huit au total) doit définir de façon cohérente les deux axes prédéfinis à partir de leurs variables structurantes respectives et, dès lors, autoriser le positionnement de chaque exploitant dans l'espace factoriel, c'est-à-dire dans l'un des quatre modèles définis.

L'application de cette démarche sur la base de données informatisées, constituée à partir de l'enquête effectuée en 1996, permet le classement suivant des 746 exploitants enquêtés (cf. graphique 1 et 2 ) :

\begin{tabular}{lcc}
\hline $\begin{array}{l}\text { Modèles de fonc tionne ment } \\
\text { des exploitations }\end{array}$ & $\begin{array}{c}\text { Nombre } \\
\text { d'expl oit ants }\end{array}$ & en \% \\
Entreprise & 197 & 26,4 \\
\hline Entreprise familiale & 178 & 23,9 \\
\hline Pays an & 167 & 22,4 \\
Transiti on ou Alternatif & 197 & 26,4 \\
\hline Non classées & 7 & 0,9 \\
Total & 746 & 100 \\
\hline
\end{tabular}


31 On remarque une assez bonne répartition des exploitations dans les quatre modèles, ce qui signifie naturellement une forte hétérogénéité des situations.

Graphique 1 : Positionnement des variables dans l'espace factoriel

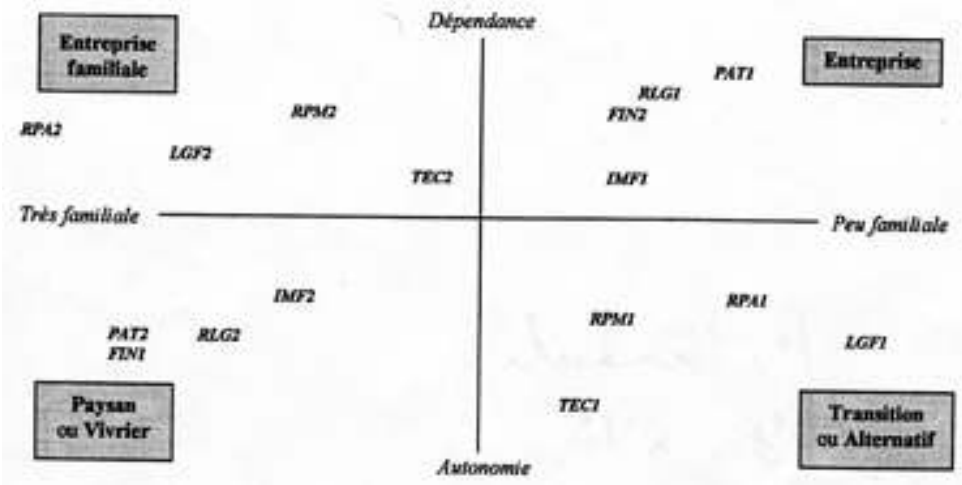

Graphique 2 : Positionnement des variables dans l'espace factoriel

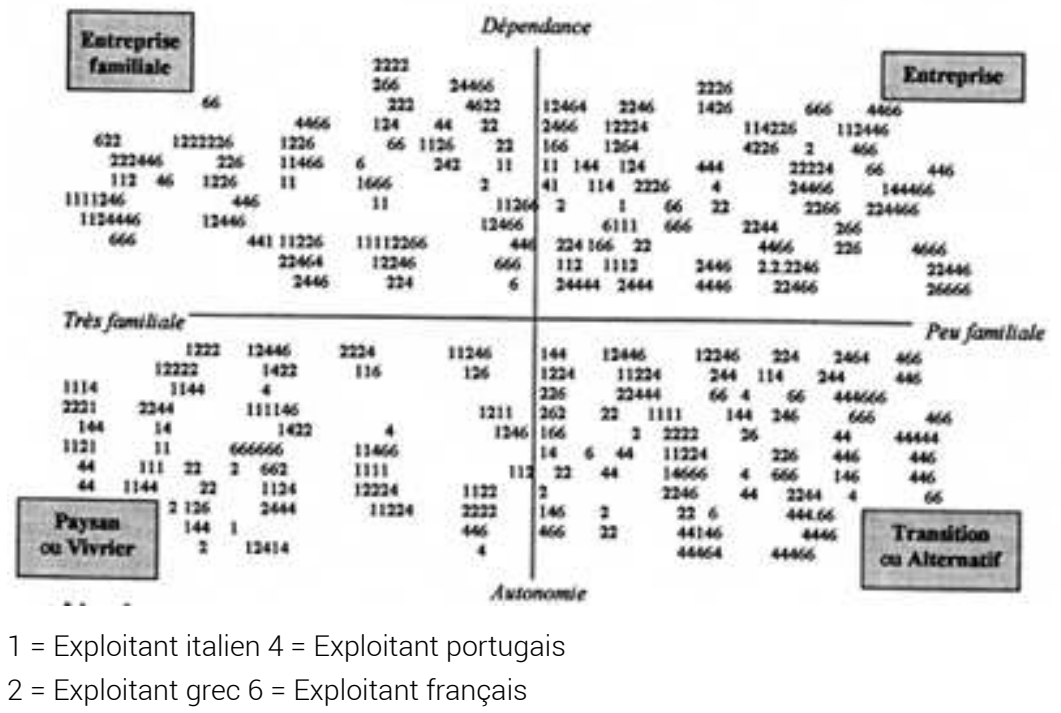

Peut-on parler d'une spécificité de l'agriculture grecque?

Pour essayer de répondre à cette question, nous avons comparé la situation grecque à celle des autres pays et situé cette comparaison à deux niveaux, celui de la réalité du fonctionnement des exploitations et celui des souhaits ou des projets formulés par ces mêmes exploitants.

La réalité

33 La comparaison de la situation concrète des exploitations donne la répartition suivante : 


\begin{tabular}{|l|c|c|c|c|}
\hline \multirow{2}{*}{ Mod èles } & \multicolumn{4}{|c|}{ Pays } \\
\cline { 2 - 5 } & Italie & Grèce & Portugal & France \\
\hline Entreprise & $18,5 \%$ & $26,9 \%$ & $26,7 \%$ & $33,3 \%$ \\
\hline $\begin{array}{l}\text { Entreprise } \\
\text { famil iale }\end{array}$ & $26,0 \%$ & $27,8 \%$ & $16,7 \%$ & $26,9 \%$ \\
\hline $\begin{array}{l}\text { Paysan } \\
\text { Transiti on }\end{array}$ & $40,4 \%$ & $24,5 \%$ & $18,1 \%$ & $10,5 \%$ \\
$\begin{array}{l}\text { ou Alternatif } \\
\text { Total }\end{array}$ & $15,1 \%$ & $20,8 \%$ & $38,6 \%$ & $29,2 \%$ \\
& $100 \%$ & $100 \%$ & $100 \%$ & $100 \%$
\end{tabular}

Les exploitants grecs, comme dans l'échantillon global, sont assez bien répartis dans les quatre modèles. Ce n'est pas le cas dans les trois autres pays; en Italie le modèle Paysan domine nettement, alors qu'au Portugal et en France la majorité des exploitations (plus de $60 \%$ ) se partagent entre les modèles Entreprise et Alternatif. En France, la majorité des exploitants enquêtés se définissent dans les modèles Entrepreneuriaux et plus du quart dans le modèle Alternatif; au Portugal, le quart des exploitants sont des Entrepreneurs, mais plus du tiers se situent dans le groupe des exploitants en Transition ou Alternatif (mais s'agit-il pour eux d'une situation transitoire en vue de rejoindre le modèle Entreprise ou plutôt de l'existence d'un véritable modèle alternatif ?) ; en Italie, la majorité des exploitants se classent dans le groupe Paysan et peu d'entre eux dans le modèle Entreprise.

Faut-il en déduire que les exploitants grecs restent encore indéterminés quant au choix d'un modèle de fonctionnement dominant? On peut en douter comme le démontre clairement l'analyse de la situation par terrain.

En effet, l'étude comparative des cinq terrains grecs étudiés montre des situations très différentes et opposées.

Ainsi le terrain de Vergina se caractérise par une très forte présence d'exploitants classés dans le modèle Transition ou Alternatif (44\%) et par une forte présence d'exploitants fonctionnant dans le modèle Entreprise (près du quart des exploitants) ; la majorité des exploitants de Thessalie $(76 \%)$, comme ceux du Péloponnèse $(60 \%)$ fonctionnent dans un modèle entrepreneurial, qu'il soit familial ou non, à la différence des exploitants béotiens qui, eux, fonctionnent plus exclusivement dans le modèle Entreprise; quant aux exploitants de la Thrace, ils sont en majorité Paysans (près de $45 \%)$ ou Entrepreneurs familiaux (37 \%).

\begin{tabular}{l|c|cccc|}
\hline \multirow{2}{*}{ Mod èles } & \multicolumn{5}{|c|}{ Terrains } \\
\cline { 2 - 6 } & Vergina & Thrace & Thessalie & Béotie & Péloponè se \\
\hline Entreprise & $24 \%$ & $6 \%$ & $28 \%$ & $51 \%$ & $30 \%$ \\
\hline $\begin{array}{l}\text { Entreprise } \\
\text { famil iale }\end{array}$ & $14 \%$ & $37 \%$ & $48 \%$ & $9 \%$ & $30 \%$ \\
\hline $\begin{array}{l}\text { Paysan } \\
\begin{array}{l}\text { Transiti on } \\
\text { ou Alternatif }\end{array}\end{array}$ & $18 \%$ & $45 \%$ & $16 \%$ & $19 \%$ & $25 \%$ \\
\hline Total & $100 \%$ & $10 \%$ & $8 \%$ & $21 \%$ & $15 \%$ \\
\hline
\end{tabular}

Sous un autre angle, on remarque que la famille reste très présente dans le fonctionnement des exploitations grecques $(52 \%$ des exploitations contre $36 \%$ en France et $34 \%$ au Portugal), mais, à la différence de l'Italie où les exploitations sont encore plus familiales (61\%), on note une répartition assez égale entre les deux modèles familiaux (Paysan et Entreprise familiale). Au niveau des terrains, comme 
précédemment, une forte différenciation apparaît : les exploitations thraciennes, très familiales (plus de $80 \%$ ), s'opposent aux exploitations béotiennes $(27 \%)$ et verginiennes (32\%), celles du Péloponnèse se situant entre ces deux extrêmes avec $55 \%$ d'exploitants identifiés comme familiaux.

Enfin, il est intéressant de noter qu'en Grèce comme en Italie, il y a beaucoup moins d'exploitants classés dans le modèle Transition ou Alternatif : 20 et $15 \%$ contre 38 et $29 \%$ au Portugal et en France. Doit-on en conclure une plus forte stabilité dans les modèles présents et une volonté nettement moins affirmée de remise en cause de ces modèles? Les projets

39 En ne considérant que les souhaits et les projets des exploitants, on obtient des résultats très différents.

\begin{tabular}{|l|cc|cc|}
\hline \multirow{2}{*}{ Modèles } & \multicolumn{4}{|c|}{ Pays } \\
\cline { 2 - 5 } Entreprise & $\begin{array}{c}\text { Itali e } \\
27,5 \%\end{array}$ & $\begin{array}{c}\text { Grèce } \\
7,1 \%\end{array}$ & $\begin{array}{c}\text { Portugal } \\
38,3 \%\end{array}$ & $\begin{array}{c}\text { France } \\
34,7 \%\end{array}$ \\
\hline Entreprise & $23,5 \%$ & $46,7 \%$ & $18,7 \%$ & $6,5 \%$ \\
famil iale & $28,2 \%$ & $36,8 \%$ & $13,9 \%$ & $12,9 \%$ \\
Pays an & $9,4 \%$ & $29,2 \%$ & $45,9 \%$ \\
Tran sition & $20,8 \%$ & $100 \%$ & $100 \%$ & $100 \%$ \\
\hline Total Arnatif & $100 \%$ & 100 & & \\
\hline
\end{tabular}

40 Contrairement à la situation précédente, on remarque une polarisation des exploitants grecs dans les deux modèles familiaux (plus de $83 \%$ ), avec une légère prédominance dans le modèle Entrepreneurial ( $+10 \%$ ). Si les Grecs souhaitent fonctionner dans un cadre familial, les exploitants italiens paraissent plus indéterminés et se répartissent assez égalitairement dans les quatre modèles. Par contre, en France comme au Portugal, le souhait des exploitants confirme leur volonté de ne pas fonctionner sur des bases familiales; en effet, $80 \%$ des exploitants français et $67 \%$ des portugais désireraient fonctionner soit dans le modèle Entreprise, soit dans le modèle Alternatif, c'est-à-dire dans les deux modèles où la famille occupe une place secondaire.

41 Notons aussi un très faible taux de représentation des exploitants grecs (7\%) dans le modèle Entreprise et cela à la différence des quatre autres pays (27,5\% en Italie, $38,3 \%$ au Portugal et 34,7 \% en France). L'agriculteur grec ne se pense pas comme un «pur » entrepreneur.

42 Notons enfin la faible représentation, aussi bien en Grèce qu'en Italie, des exploitants dans le modèle en Transition ou Alternatif: 9 et $20 \%$ des exploitants alors qu'en France et au Portugal le taux est respectivement de 46 et $29 \%$. Cela confirme la relative stabilité des exploitants grecs et italiens dans les modèles familiaux et leur peu de volonté de les remettre en cause, ce qui est loin d'être le cas surtout parmi les exploitants français, mais aussi portugais. 


\begin{tabular}{|lc|cc|cc|}
\hline \multirow{2}{*}{ Modèles } & \multicolumn{5}{c|}{ Terrains } \\
\cline { 2 - 6 } Entreprise & $\begin{array}{c}\text { Vergina } \\
6 \%\end{array}$ & $\begin{array}{c}\text { Thrace } \\
6 \%\end{array}$ & $\begin{array}{c}\text { Thessalie } \\
4 \%\end{array}$ & $\begin{array}{c}\text { Béotie } \\
14 \%\end{array}$ & $\begin{array}{c}\text { Péloponè se } \\
5 \%\end{array}$ \\
\hline $\begin{array}{l}\text { Entreprise } \\
\text { famil iale }\end{array}$ & $40 \%$ & $53 \%$ & $50 \%$ & $51 \%$ & $30 \%$ \\
Paysan & $46 \%$ & $37 \%$ & $38 \%$ & $21 \%$ & $45 \%$ \\
$\begin{array}{l}\text { Transition } \\
\text { ou Alternatif }\end{array}$ & $8 \%$ & $4 \%$ & $8 \%$ & $14 \%$ & $20 \%$ \\
\hline Total & $100 \%$ & $100 \%$ & $100 \%$ & $100 \%$ & $100 \%$ \\
\hline
\end{tabular}

Le souhait fortement exprimé de fonctionner sur des bases familiales se confirme, sans exception, sur les cinq terrains grecs étudiés : $86 \%$ des exploitants de Vergina, $89 \%$ des exploitants de Thrace, $88 \%$ des thessaliens, $71 \%$ des béotiens et $75 \%$ des exploitants du Péloponnèse ; rappelons que, dans l'échantillon global, seulement $52 \%$ des exploitants enquêtés se pensent dans un système de fonctionnement familial.

Néanmoins, la Béotie se distingue très nettement des autres régions par le fait que $14 \%$ des exploitants souhaitent devenir des entrepreneurs ; et même si ce taux peut paraître particulièrement faible, il l'est encore plus sur les quatre autres terrains grecs : $6 \%$ à Vergina et en Thrace, 5 et $4 \%$ dans le Péloponnèse et en Thessalie.

De la réalité au projet

Le croisement de la situation des exploitants grecs (réalité) avec leurs espérances (projets) permet d'évaluer avec plus de précisions leur niveau de satisfaction: fonctionnent-ils ou non dans le modèle qu'ils espèrent ? Sinon, par rapport au modèle dans lequel ils sont, dans quels autres modèles souhaiteraient-ils fonctionner?

On obtient le tableau suivant :

\begin{tabular}{|c|c|c|c|c|c|c|}
\hline \multicolumn{7}{|c|}{ RÉALITÉ } \\
\hline & & Entreprise & $\begin{array}{l}\text { Entreprise } \\
\text { familiale }\end{array}$ & Paysan & $\begin{array}{c}\text { Transition } \\
\text { ou Alternatif }\end{array}$ & Total \\
\hline \multirow{5}{*}{$\begin{array}{l}P \\
R \\
O \\
J \\
E \\
T\end{array}$} & Entreprise & $16 \%$ & $\begin{array}{r}14 \% \\
3 \%\end{array}$ & & $\begin{array}{l}33 \% \\
12 \%\end{array}$ & $100 \%$ \\
\hline & $\begin{array}{l}\text { Entreprise } \\
\text { faniliale }\end{array}$ & $49 \% \quad 26 \%$ & $54 \%$ & $40 \% \quad 21 \%$ & $46 \%$ & $100 \%$ \\
\hline & Paysan & $21 \%$ & $36 \% \quad 27 \%$ & $52 \%$ & $30 \% \quad 17 \%$ & $100 \%$ \\
\hline & $\begin{array}{l}\text { Transition } \\
\text { ou Alternatif }\end{array}$ & $14 \%$ & $7 \% \quad 20 \%$ & $8 \% \quad 20 \%$ & $12 \%$ & $100 \%$ \\
\hline & Total & $100 \%$ & $100 \%$ & $100 \%$ & $100 \%$ & \\
\hline
\end{tabular}

Ces résultats appellent plusieurs observations : c'est dans les deux modèles familiaux (Entreprise et Paysan) qu'il y a le plus grand nombre d'exploitants en cohérence entre ce qu'ils sont et ce qu'ils projettent. $54 \%$ des Entrepreneurs familiaux se projettent dans ce même modèle et $52 \%$ des Paysans se pensent dans ce même modèle Paysan. Cela confirme le fort attachement des agriculteurs grecs à la famille.

Ce n'est pas le cas pour les exploitants identifiés comme Entrepreneurs : seulement $16 \%$ d'entre eux se projettent dans ce même modèle. Il en est de même pour les autres exploitants; aucun des Paysans ne se projette dans le modèle Entreprise et seulement $3 \%$ des Entrepreneurs familiaux et $12 \%$ de ceux classés dans le modèle en Transition ou Alternatif. Il apparait clairement que le modèle Entreprise attire assez peu les exploitants 
grecs et qu'il n'est donc pas pour eux le modèle de référence, comme c'est ou ce fut le cas dans d'autres pays.

Cette importance donnée à la famille dans le fonctionnement de l'exploitation ne doit pas être interprétée comme une volonté de se maintenir dans la tradition et un refus du progrès ; en effet, la majorité des exploitants qui ne sont pas en cohérence se projettent plutôt dans le modèle Entreprise familiale que dans le modèle Paysan. Cet attachement à la famille des exploitants grecs n'est donc pas un refus de la modernité, mais semble plutôt s'inscrire dans une autre forme de modernité.

Notons, enfin, le peu de succès rencontré par le modèle Transition ou Alternatif: $14 \%$ parmi les Entrepreneurs, seulement 7 et $8 \%$ parmi les Entrepreneurs familiaux et les Paysans. L'exploitant grec ne se pense, pour l'avenir, ni dans une dynamique de transition, ni dans autre modèle. Ce constat, ajouté à celui du refus par une très forte proportion d'agriculteurs de fonctionner dans le modèle Entreprise, confirmerait l'hypothèse avancée précédemment selon laquelle l'agriculture grecque, contrairement aux autres, s'organise et cherche encore plus à s'organiser dans des modèles familiaux.

On constate peu de différence au niveau des terrains : le modèle familial est fortement présent partout. Par contre, une certaine divergence apparaît en fonction de la répartition des exploitants dans l'un ou l'autre des deux modèles familiaux (Entreprise familiale ou Paysan); ainsi l'agriculture du Péloponnèse semble plus fortement ancrée dans le modèle Paysan, alors que celles de Thessalie et surtout de Béotie paraissent plutôt séduites par le modèle Entrepreneurial, les Thraciens et les Verginiens restant partagés.

Cette analyse comparative montre clairement une spécificité de l'agriculture grecque en ce sens que, contrairement aux autres pays étudiés, elle reste (et souhaite rester) principalement organisée autour de la famille et que, à la différence de l'Italie, cette agriculture se pense dans une modernité très conforme aux normes européennes préconisées et encouragées.

Ceci étant, une étude qualitative doit être effectuée, en complément de cette approche comparative et quantitative, en vue d'analyser avec plus de précision les termes de cette spécificité qui, probablement, ne peut se comprendre uniquement dans le cadre des rapports sociaux de production agricole, mais doit se situer dans le cadre plus large des activités non agricoles, du marché du travail (emploi et chômage) et de la nature des rapports ville/campagne.

\section{NOTES}

1. Mendras H. La fin des paysans, Armand Colin, U2, 1970, rééd. Actes sud, 1991. Gervais M., Jollivet M. et Tavernier Y., L'histoire de la France rurale, tome IV, Paris, Seuil, 1976.

2. Réseau Européen d'Analyse Locale Comparée des Exploitations Agricoles.

3. Pour plus d'informations, se reporter aux tomes 1 (p. 9-28) et 2 (p. 49-58) de L'agriculture familiale, Hugues Lamarche, (coord.), L'Harmattan, Paris, 1992 et 1994. 


\section{RÉSUMÉS}

À partir d'une enquête effectuée dans quatre pays européens (l'Italie, le Portugal, la Grèce et la France), l'auteur tente dans une démarche comparative de répondre à cette délicate question de savoir s'il y a un modèle grec d'exploitations agricoles. Dotée d'une grille théorique d'analyse des modèles de fonctionnement des exploitations agricoles, il montre la spécificité des agriculteurs grecs, tant du point de vue de leur situation présente que des projets qu'ils souhaitent pour leur avenir. Cette analyse, bien que réalisée à partir de cinq études locales exemplaires de la diversité de l'agriculture grecque, ne fait apparaître que très peu de différence au niveau des terrains ; cela autorise à une certaine généralisation des résultats. L'exploitation grecque reste (et souhaite rester) principalement organisée autour de la famille et se pense dans une modernité qui apparaît très conforme aux nouvelles orientations de la politique européenne.

Based on research carried out in four European countries (Italy, Portugal, Greece and France), the author attempts to answer the delicate question of whether there is a Greek model of farming through comparison. Equipped with a theoretical framework of analysis of models of functioning farms, he demonstrates the specificity of Greek farmers, with regard to both their current situation and their future plans. Despite being drawn from five local studies characteristic of the diversity of Greek agriculture, this analysis reveals little difference between the areas. It allows for some generalisation of the results. Greek farming remains (and wishes to remain) organised mainly through the family unit and maintains its position in a modernity which seems compatible with new directions in European policy.

\section{AUTEUR}

HUGUES LAMARCHE

Ladyss, Cnrs 